\title{
An upright eyedrop bottle: accuracy, usage of excess drops, and contamination compared to a conventional bottle
}

This article was published in the following Dove Press journal:

Clinical Ophthalmology

28 July 2016

Number of times this article has been viewed

\author{
Isaiah J Davies' \\ Ninita H Brown ${ }^{2}$ \\ Joanne C Wen ${ }^{3}$ \\ Sandra S Stinnett ${ }^{1}$ \\ Kelsey Kubelick ${ }^{4,5}$ \\ Roma P Patel ${ }^{6}$ \\ Kristin L Benokraitis ${ }^{7}$ \\ Latoya Greene' \\ Curry Cheek' \\ Kelly W Muir ${ }^{1,8}$ \\ 'Department of Ophthalmology, \\ Duke University School of Medicine, \\ Durham, NC, USA; ${ }^{2}$ Metrolina Eye \\ Associates, Charlotte, NC, USA; \\ ${ }^{3}$ Department of Ophthalmology, \\ University of Washington, Seattle, WA \\ USA; ${ }^{4}$ Wallace H Coulter Department \\ of Biomedical Engineering, Georgia \\ Institute of Technology, ${ }^{5}$ Wallace $\mathrm{H}$ \\ Coulter Department of Biomedical \\ Engineering, Emory University School \\ of Medicine, Atlanta, Georgia, USA; \\ 'University of California Davis Health \\ System Eye Center, Sacramento, CA, \\ USA; ${ }^{7} E G-G I L E R O$, Durham, NC, \\ USA; ${ }^{8}$ Health Services Research \& \\ Development, Durham VA Medical \\ Center, Durham, NC, USA
}

Correspondence: Kelly W Muir Duke University School of Medicine, Duke University Medical Center, Box 3802, Durham, NC 277I0, USA

$\mathrm{Tel}+\mathrm{I} 9196843283$

Fax + I 9196818267

Email kelly.muir@duke.edu
Purpose: This study tested the feasibility of using an upright eyedrop bottle (UEB), a device designed to assist patients with eyedrop placement without reclining their head.

Patients and methods: Experienced eyedrop users were enrolled who answered "yes" to the question, "Do you ever have trouble getting your eyedrops in?" After being shown a multimedia presentation and answering a questionnaire regarding eyedrop usage, participants were observed instilling eyedrops. Participants were instructed to instill a single eyedrop in each eye with both a standard bottle and the UEB. They repeated this process three times. With each trial, the amount of time taken to instill drops was recorded, as well as whether a drop landed in the eye (accuracy), if excess drops were used, and if the bottle tip was contaminated.

Results: Forty participants were enrolled, with an average age of $72.4 \pm 8.9$ years; the majority were females ( 24 females). Thirty-four participants had been using eyedrops for at least 1 year. The time required to instill eyedrops was significantly less with the UEB in the second and third trials. There was no difference in accuracy between the conventional bottle and the UEB in the left or right eye in any trials. Significantly more participants used excess number of drops while using the conventional bottle in both the left and right eyes in all three trials. The bottle tip was never contaminated with the UEB. Depending on the trial and the eye, the conventional bottle was contaminated by between $42 \%$ and $53 \%$ of participants.

Conclusion: The UEB has the potential to assist patients with eyedrop placement. Although there was no difference in accuracy between the UEB and the conventional bottle, the UEB was associated with less use of excess drops and less contamination of the bottle tip, compared to the conventional bottle.

Keywords: glaucoma, compliance, medical devices

\section{Introduction}

It is well known that patients struggle with the physical instillation of eyedrops. As many as $25 \%$ of patients who instill their own drops were unable to place a drop in their eye during a trial of drop instillation. ${ }^{1}$ When patients with visual impairment are taken into account, this percentage has been reported to be as high as $30 \%{ }^{2}$ In addition to struggling to place a drop in the eye, as many as $59 \%$ of patients have been observed to express multiple drops from the bottle in an attempt to get only one drop into the eye. ${ }^{1}$ Furthermore, $80 \%$ of patients have been observed to make contact between the tip of the eyedrop bottle and the eye. ${ }^{3}$ Defining successful eyedrop instillation as placing one drop in the conjunctival cul-de-sac without contaminating the tip of the bottle, one study found as few as $8.5 \%$ of patients to be successful. ${ }^{4}$ Therefore, for many glaucoma patients who administer eyedrops, there is room for improvement 
in the arenas of efficiency, medication conservation, and contamination.

A patient's inability to successfully instill an eyedrop can have multiple consequences. If a patient is unable to actually place a drop in the eye, their disease will not be treated. If they place too many drops, the consequences can range from systemic side effects ${ }^{5}$ to increased expenses. The tip is often the most contaminated portion of eyedrop bottles. ${ }^{6-8}$ There have been multiple reports of eye infections that were associated with contaminated bottles. ${ }^{9-11}$ This leads to the possibility of eyedrops causing infection when instilled incorrectly. There have also been reports of inadvertent trauma from the tip of an eyedrop bottle, leading to conjunctival inflammation. ${ }^{12}$

One barrier that patients have reported in administering eyedrops is arthritis. ${ }^{13}$ Cervical osteoarthritis can limit a person's ability to recline the head. Although there are several commercially available eyedrop aids that assist with aiming and squeezing the bottle, all require that the patient recline the head. We have developed an upright eyedrop bottle (UEB) ${ }^{14}$ to prevent the necessity of reclining the head. In an Institutional Review Board (IRB)-approved study with a small cohort of patients, the original device was tested for feasibility of use and to gather feedback. Based on the observations of the study team and feedback from participants, three subsequent prototypes were developed, each modified in an iterative process of design refinement and testing.

The purpose of this study was to test the feasibility of use of the most recent iteration of the UEB (Figure 1) among experienced eyedrop users. Secondary aims included studying bottle tip contamination rates and rates of excess drop usage using the UEB.

\section{Methods}

Participants were recruited from a single academic glaucoma clinic from May 2014 to March 2015 in this Duke Medicine Institutional Review Board-approved study. Included participants were at least 18 years of age, prescribed eyedrops for at least 6 months, reported regular self-administration of eyedrops, and answered "yes" to the question, "Do you ever have trouble getting your eyedrops in?" Potential participants were excluded for best-corrected visual acuity $<20 / 400$ in both eyes and history of intraocular surgery within the preceding month. After giving written informed consent, participants were shown a multimedia presentation demonstrating the proper use of both the UEB as well as a standard eyedrop bottle. Following the presentation, participants filled out a questionnaire about their home eyedrop usage.
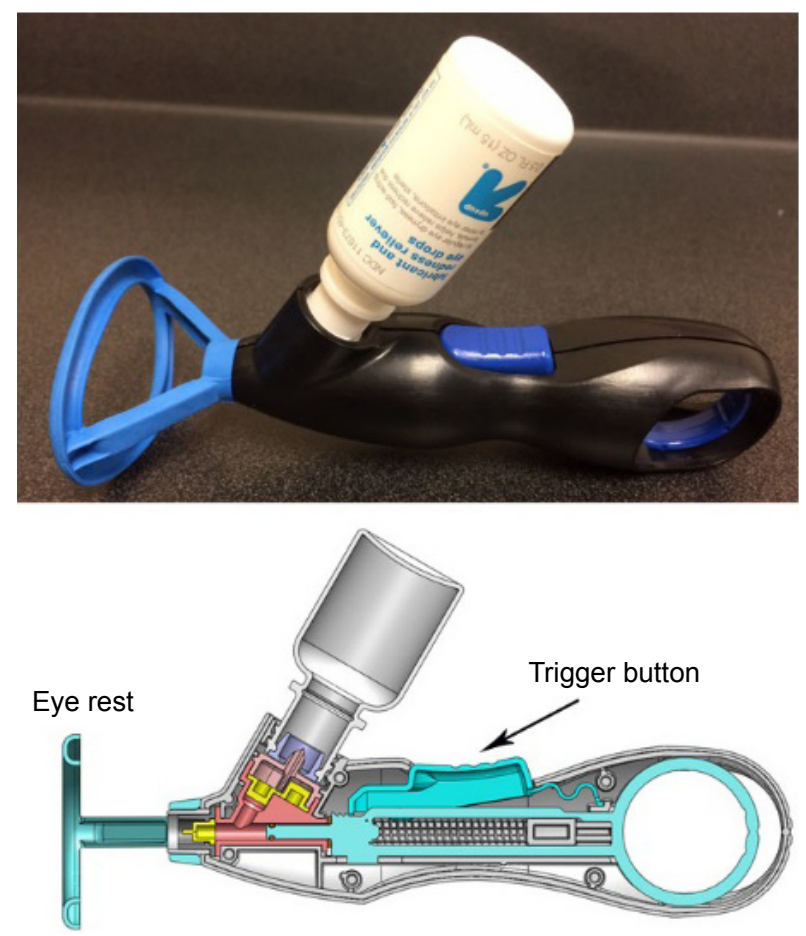

Figure I The UEB, with a bottle of artificial tears attached, is depicted in the upper portion of the figure. A schematic of the bottle design is found in the lower portion of the figure.

Abbreviation: UEB, upright eyedrop bottle.

Following the questionnaire, participants were taken to an exam room with an examination chair, mirror, sink, and tissues. Also in the room were two, closed sterile bottles containing saline solution, the UEB, and a trained observer to record the participant's results. The participants were randomized as to whether they would use the standard eyedrop bottle or UEB first. Each participant was instructed to place one drop in each eye from the first bottle, and afterward to place one drop in each eye from the second bottle. Each participant repeated this sequence three times. The observer recorded 1) time required for instillation, 2) if the participant placed at least one drop within the eye (accuracy), 3) if fluid rolled down the cheek indicating excess drops were used, and 4) if the participant contaminated the tip of the bottle by touching the eye or surrounding surfaces.

Descriptive statistics were derived, including mean, median, range, and standard deviation. For the outcome of time to instillation of an eyedrop, bottle types were compared with the Wilcoxon signed-rank test. The McNemar's test for difference in paired proportions was used to test the difference between bottle types for the outcomes of accuracy, excess drop use, and contamination of the bottle. For all comparisons, a $P$-value of $<0.05$ was considered statistically significant. 


\section{Results}

In total, 40 participants were enrolled in this study. As illustrated in Table 1, the average age of participants was $72.4 \pm 8.9$ years, with the majority of participants being female and right-handed. More than half of the participants had visual acuity better than 20/40 in at least one eye, and $85 \%$ of the participants in the study had been using eyedrops for more than 1 year.

As shown in Table 2, the time required to instill drops with the UEB was shorter than with the conventional bottle

Table I Participant characteristics

\begin{tabular}{|c|c|}
\hline Demographics & $\mathbf{N}(\%)$ \\
\hline Number of participants enrolled & 40 \\
\hline Average age \pm standard deviation (years) & $72.4 \pm 8.9$ \\
\hline Age range (years) & $55-87$ \\
\hline Females:males & 24 (60): $16(40)$ \\
\hline Right-handed:left-handed & $35(87.5): 5(12.5)$ \\
\hline in both eyes & $16(40)$ \\
\hline $\begin{array}{l}\text { Visual acuity worse than } 20 / 60 \\
\text { in better seeing eye }\end{array}$ & $9(22.5)$ \\
\hline \multicolumn{2}{|l|}{ Survey responses } \\
\hline \multicolumn{2}{|l|}{ Duration of eyedrop use } \\
\hline$>2$ years & $32(80)$ \\
\hline $\mathrm{I}-2$ years & $2(5)$ \\
\hline $7-12$ months & $4(10)$ \\
\hline $2-6$ months & $2(5)$ \\
\hline \multicolumn{2}{|l|}{ Eyedrops prescribed for } \\
\hline Both eyes & $29(72.5)$ \\
\hline Right eye only & $7(17.5)$ \\
\hline Left eye only & $4(10)$ \\
\hline \multicolumn{2}{|l|}{ Total number of drops } \\
\hline \multicolumn{2}{|l|}{ prescribed per day } \\
\hline$\leq 2$ & I5 (37.5) \\
\hline $3-5$ & $14(35)$ \\
\hline$\geq 6$ & I I (27.5) \\
\hline \multicolumn{2}{|l|}{ Person who taught proper technique } \\
\hline Nurse/technician & $7(\mid 7.5)$ \\
\hline Ophthalmologist & $10(25)$ \\
\hline Instruction sheet & $2(5)$ \\
\hline Self-teaching & $21(52.5)$ \\
\hline \multicolumn{2}{|l|}{ Hardest part of drop placement } \\
\hline Aiming the bottle & $27(67.5)$ \\
\hline Keeping eyes open & $10(25)$ \\
\hline Squeezing out the drop & $7(17.5)$ \\
\hline Tipping head & $2(5)$ \\
\hline Opening the bottle & $2(5)$ \\
\hline Other $^{\mathrm{a}}$ & $4(10)$ \\
\hline \multicolumn{2}{|l|}{ How often do you miss your eyedrops? } \\
\hline I-2 times/d & $16(40)$ \\
\hline$\leq 4$ times/wk & $12(30)$ \\
\hline Never & $12(30)$ \\
\hline
\end{tabular}

Notes: aSpecific responses: "cannot administer without mirror", "none", "never one drop", "squeezing out the bottle, bottle too small, using hand with arthritis, squeezing out more than needed". in all three trials, with statistical significance in the last two trials.

As noted in Table 3, there was neither statistical significance nor pattern of differences in the number of participants able to instill a drop in their left or right eye across all three trials. Fluid rolled down the cheek significantly less with the UEB than the conventional bottle in all three trials of both eyes. Finally, the tip of the UEB was not contaminated in any trial by either eye; the tip was contaminated by $16-20$ patients per trial in all three trials with the conventional bottle.

\section{Discussion}

The purpose of this study was to address the feasibility of use of the UEB for eyedrop placement as well as to understand the rates of contamination and excess eyedrop use with the UEB.

\section{Feasibility}

There was no statistically significant difference in accuracy of drop instillation between the conventional eyedrop bottle and UEB. Across all three trials and both eyes, approximately $70 \%-90 \%$ of participants were able to accurately place a drop with both the conventional bottle and UEB. The ability to instill at least one drop in the eye in this study was within the same range reported by many other studies. ${ }^{1-3,15-18}$ It is interesting that accuracy with the conventional bottle in this study was similar to other studies given that participants were specifically selected because they admitted to having trouble with self-administration of their eyedrops. They were not specifically asked what aspects of self-administration they struggled with and were not objectively screened for trouble instilling eyedrops either. These participants may have struggled more with other aspects of drop placement, rather than accuracy, which may explain why the UEB did not improve accuracy in this particular group of participants.

Although the UEB did not improve accuracy, it did not decrease accuracy either. The majority of participants in the study (34/40) had been taking drops for over a year, and thus had the opportunity to practice with the conventional bottle for over a year. The UEB was not known to patients prior to this study, and yet it still performed as well as the conventional bottle. In addition to performing as well as the conventional bottle, the UEB allowed participants to place their eyedrops faster than with the conventional bottle. Curiously, the time to instill drops decreased in each consecutive trial with not only the UEB but also the conventional bottle. It is possible that the conventional bottle used in the study differed from the one used at home (ie, different shape, 
Table 2 Time $^{\text {a }}$ required to instill eyedrops in both eyes with conventional bottle and UEB

\begin{tabular}{|c|c|c|c|c|c|}
\hline Trial $^{\mathbf{b}}$ & Statistic & Conventional bottle & UEB & Difference & $P$-value \\
\hline \multirow[t]{5}{*}{ I } & $\mathrm{N}$ & 40 & 40 & 40 & 0.496 \\
\hline & Mean (standard deviation) & $40(15.3)$ & $38.8(16.4)$ & $-1.8(12.3)$ & \\
\hline & Minimum & 22 & 16 & -45 & \\
\hline & Median & 37 & 36 & -1 & \\
\hline & Maximum & 91 & 91 & 21 & \\
\hline \multirow[t]{5}{*}{2} & $\mathrm{~N}$ & 39 & 40 & 39 & 0.004 \\
\hline & Mean (standard deviation) & $31.4(\mid 1.6)$ & $29.1(12.6)$ & $-3.3(9.9)$ & \\
\hline & Minimum & 16 & 15 & -24 & \\
\hline & Median & 29 & 25 & -4 & \\
\hline & Maximum & 57 & 63 & 24 & \\
\hline \multirow[t]{5}{*}{3} & $\mathrm{~N}$ & 40 & 40 & 40 & 0.001 \\
\hline & Mean (standard deviation) & $28.9(9.9)$ & $26.3(11.1)$ & $-4.2(9.6)$ & \\
\hline & Minimum & 14 & 13 & -22 & \\
\hline & Median & 27 & 25 & -5 & \\
\hline & Maximum & 48 & 71 & 27 & \\
\hline
\end{tabular}

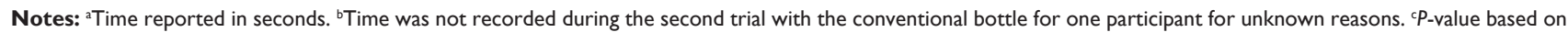
Wilcoxon signed-rank test of median difference equal to zero, $P$-value $<0.05$ considered statistically significant and is denoted in bold.

Abbreviation: UEB, upright eyedrop bottle.

size, or stiffness), and thus participants got faster as their comfort level with the bottle increased. Successful instillation of drops with the UEB with less time but not loss of accuracy was a surprising finding given that participants were using the UEB for the first time.

\section{Excess drops}

Significantly fewer participants used excess drops with the UEB as compared to the conventional eyedrop bottle across all trials with both eyes. The percentage of participants using excess drops ranged from $63 \%$ to $74 \%$ with the conventional bottle and from $24 \%$ to $41 \%$ with the UEB. The percentage of participants expelling excess drops from the conventional bottle was slightly more than what Stone et $\mathrm{al}^{1}$ found. Their research showed that up to $59 \%$ of patients used either more than one drop or instilled a "stream of fluid". ${ }^{1}$ Kass et al ${ }^{19}$ observed that $48 \%$ of patients used more than one drop. Brown et $\mathrm{al}^{3}$ recorded $21 \%$, Sleath et $\mathrm{al}^{15}$ found $30 \%$, and Hennessy et $\mathrm{al}^{16}$ observed $22 \%$ of patients used greater than one drop. Kass et $\mathrm{al}^{19}$ did find that the number of excess drops used correlated with increasing patient age. This may partially explain why more participants used excess drops from the conventional bottle than previous studies. The average age of participants was $72.4 \pm 8.9$ years, older than the average age in any of the previously mentioned studies where fewer patients used excess drops. Given that glaucoma is more prevalent with advanced age, the age cohort of this study may represent the challenges faced with glaucoma eyedrop instillation more appropriately than studies with younger patients. In addition, participants were specifically selected

Table 3 Comparing accuracy, excess drops, and contamination between conventional bottle and UEB

\begin{tabular}{|c|c|c|c|c|c|c|c|}
\hline \multirow[t]{2}{*}{ Observed parameter } & \multirow[t]{2}{*}{ Trial } & \multicolumn{3}{|l|}{ Right eye $\left(\mathbf{N}=39^{a}\right)$} & \multicolumn{3}{|l|}{ Left eye $\left(\mathbf{N}=\mathbf{3 8}^{\mathrm{b}}\right)$} \\
\hline & & Conventional bottle, n (\%) & UEB, n (\%) & $P$-value ${ }^{c}$ & Conventional bottle, n (\%) & UEB, n (\%) & $P$-value \\
\hline At least one drop was & 1 & $29(74)$ & $28(72)$ & 0.781 & $33(87)$ & $31(82)$ & 0.527 \\
\hline \multirow[t]{2}{*}{ placed into the eye } & 2 & $32(82)$ & $35(90)$ & 0.257 & $28(76)$ & $32(84)$ & 0.405 \\
\hline & 3 & $35(90)$ & $36(92)$ & 0.705 & $34(89)$ & $34(89)$ & 1.000 \\
\hline \multirow[t]{3}{*}{ Excess drops were used } & 1 & $27(69)$ & $16(4 I)$ & 0.022 & $27(7 I)$ & II (29) & 0.002 \\
\hline & 2 & $29(74)$ & $12(3 \mid)$ & 0.001 & $28(74)$ & $9(24)$ & $<0.001$ \\
\hline & 3 & $29(74)$ & $12(3 I)$ & 0.001 & $24(63)$ & $13(34)$ & 0.016 \\
\hline Bottle tip was & 1 & $19(49)$ & $0(0)$ & - & $20(53)$ & $0(0)$ & - \\
\hline \multirow[t]{2}{*}{ contaminated } & 2 & $20(5 I)$ & $0(0)$ & - & $16(42)$ & $0(0)$ & - \\
\hline & 3 & $19(49)$ & $0(0)$ & - & $19(50)$ & $0(0)$ & - \\
\hline
\end{tabular}

Notes: ${ }^{a}$ One participant with visual impairment in the right eye did not attempt drop placement in right eye with conventional bottle or UEB. ${ }^{\mathrm{b}} \mathrm{One}$ participant with visual impairment in the left eye and one participant with left-sided ocular prosthesis did not attempt drop placement in left eye with conventional bottle or UEB. ${ }^{c}$-value based on McNemar's test for difference in paired proportions, $P$-value $<0.05$ is considered statistically significant and denoted in bold. The - represents not applicable values. Abbreviation: UEB, upright eyedrop bottle. 
for the study if they had difficulty with self-administration, which may include trouble applying only one drop. Finally, saline was used in both the conventional bottle and UEB in this study, which may have a lower viscosity than glaucoma medications, leading to a higher likelihood of it dribbling out of the eye.

Less use of excess drops with the UEB compared to the conventional bottle may be related to the metered dose mechanism of delivery. The UEB has been designed with the intent that the volume that enters the fluid chamber of the device is the volume delivered. By pushing a button to deliver medication, patients deliver the same amount of medicine with every push. This is in contrast to squeezing a standard eye bottle, where a patient must estimate how much force to apply to expel a single drop. In addition, different bottles require different amounts of force, ${ }^{20}$ and as Winfield et $\mathrm{al}^{21}$ found in their sample of patients, up to $13 \%$ cannot even generate enough force to expel one drop from a bottle. ${ }^{21}$ This difference in force may be due to bottle characteristics such as the dimensions, shape, wall thickness, or even bottle material. While the amount of force required to deliver medication was not tested with the UEB, it is possible that the metered dose mechanism may even allow patients who cannot squeeze a conventional bottle to deliver their own eyedrops.

A possible consequence of excess drops is systemic side effects. Examples of such side effects include bradycardia and bronchospasm due to ophthalmic solutions of $\beta$-blockers. ${ }^{5}$ Using excess drops can also lead to increased costs for patients. When patients use too many drops, this may cause them to run out of drops earlier than anticipated, leading to refills of medication that would otherwise be unnecessary, or running out of medication and being unable to obtain a refill prior to the authorized refill date.

\section{Contamination}

The most obvious area where the UEB differed from conventional eyedrop bottles was in contamination rates of the tip of the bottle. The UEB had no contamination events. This is remarkable considering contamination rates for the conventional bottle in this study ranged from $42 \%$ to $53 \%$. The contamination rates for the conventional bottle were similar to those found in other studies. ${ }^{19,22}$ The results suggest that the UEB will lower contamination rates. This is because the UEB design employs a physical barrier that keeps the tip of the bottle from touching the eye or its surrounding structures. Furthermore, the device is designed to be disposable after a single use, meaning the device does not need to be cleaned after use.
One limitation of the prototype used in the study was that in order for the UEB to accept the conventional bottle, the tip of the conventional bottle needed to be popped off. This process has the potential to lead to contamination. In order to address this, a design has been developed that does not require the tip of the bottle to be removed. In addition to this limitation, the device cannot be used with single-use containers.

One might speculate that patients will contaminate the tip of a conventional bottle less with experience, negating the need for a device to assist with this issue. Schwartz et $\mathrm{al}^{23}$ attempted to see if patients improved their eyedrop placement technique over a 12 -week period. At the start of the 12 -week period, $18.2 \%$ of patients contaminated the bottle compared to $18.5 \%$ at the end of the 12 -week period. ${ }^{23}$ Although the present study only observed participants over the course of three trials, the results agree with Schwartz et al. ${ }^{23}$ Contamination rates with the conventional bottle did not show a decreasing trend as trials progressed. Also important to note is that the majority (34/40) of the participants in this study had been using their drops for over a year and thus had experience already. This is in comparison to their lack of experience with the UEB, and yet they still did not contaminate the tip of the UEB bottle.

Contaminated medications do have the potential to cause clinical consequences. Schein et $\mathrm{al}^{8}$ found that when patients' conjunctiva were cultured for microorganisms, 34\% of patients using topical ocular medications grew potentially pathogenic organisms compared to only $10 \%$ of patients who were not using medications. The same study found that $42 \%$ of patients had at least one of their medications contaminated, and of those patients with contaminated medications, $60 \%$ of them had the same organisms cultured from both their conjunctiva and their medication. Also, of those $42 \%$ of patients with a contaminated medication, $45 \%$ of them had a potentially pathogenic organism cultured from the medication. An interesting observation from this study was that when a patient had a potentially pathogenic organism grow from both their conjunctival and medication cultures, it was always a gram-negative organism. ${ }^{8}$ However, one must note that there are similar studies with different results. Geyer et $\mathrm{al}^{7}$ found no significant difference in positive conjunctival cultures between patients who were and were not using topical ocular medications. In that study, $28 \%$ of the medications had positive cultures, and of those, $22 \%$ had gram-negative organisms. None of the potentially pathogenic organisms were found in both the conjunctiva and medication of the same patient. It is interesting though, that 
in that study, 37 out of 37 patients who stated they touched their eyes while placing drops had at least one medication that was contaminated. ${ }^{7}$

While Geyer et $\mathrm{al}^{7}$ and Schein et $\mathrm{al}^{9}$ discuss the percentage of medications and conjunctiva with potentially pathogenic organisms, there have also been reports of eye infections associated with contaminated bottles. ${ }^{10,11}$ This is in addition to reports of inadvertent trauma leading to conjunctival inflammation. ${ }^{12}$ The UEB was able to decrease the number of tip contacts between the dropper and eye to zero, thus hopefully negating any degree of infection or inflammation that might occur as a result of tip contact.

\section{Other devices}

Other innovations and devices have attempted to address the various aspects of eyedrop placement that patients struggle with. It is difficult to compare the performance of the UEB to these devices as information varies in the literature, but it is informative to understand examples of other eyedrop aids. Two such devices are the Opticare ${ }^{\circledR}$ eyedrop dispenser (Cameron Graham Limited, Huddersfield, UK) and Xal-Ease ${ }^{\circledR}$ (Pfizer Ophthalmics, New York, NY, USA).

The Opticare ${ }^{\circledR}$ eyedrop dispenser was designed to assist patients with conditions such as arthritis in squeezing their eyedrop bottles. It also serves to improve aim and decrease contamination. When studied, it objectively improved patients' ability to squeeze drops from a bottle from $48 \%$ to $90 \%$ and improved patients' ability to get a drop into the eye with mild or no difficulty from $48 \%$ to $86 \%{ }^{24}$ It is however important to note that this device was studied in patients with rheumatoid arthritis. It would be interesting to see if improvements in accuracy were observed in patients without rheumatoid arthritis. One similarity between Opticare ${ }^{\circledR}$ and the UEB was that none of the patients contaminated the bottle with both Opticare ${ }^{\circledR 24}$ and the UEB. Subjectively, patients felt they were better able to squeeze and aim the bottle and dispense the proper amount of drops with Opticare ${ }^{\circledR}$, but no objective measure of drops dispensed was presented. ${ }^{24}$

Xal-Ease ${ }^{\circledR}$ delivery device was designed to help align the bottle over the eye and deliver only one drop using a button instead of squeezing the bottle. The device was only studied subjectively, but it was found that with the device patients felt they were less likely to need help with drop instillation. In addition, with the device, patients felt they contaminated the tip less (decreased from $35.6 \%$ to $3.2 \%$ ), had increased accuracy (increased from $49.9 \%$ to $62.4 \%$ ), and delivered less excess drops. Overall, $70 \%$ of patients were satisfied with the device. Important to note is that this device is only compatible with specific branded medications. ${ }^{25}$

\section{Limitations}

This study had several limitations in addition to those already mentioned in the "Discussion" section. Participants were recruited from a single academic institution, and so it is possible that a different study population may have shown different results. In addition, this was a small study with a convenience sample. Study participants were asked to instill their eyedrops in an artificial setting as they were in a clinic and possibly using bottles that were different from those they used on a daily basis. Eyedrop administration was scored by a trained observer but not videotaped, which would have allowed review to ensure fidelity.

\section{Conclusion}

Up to $78 \%$ of patients welcome the idea of a compliance aid to assist in eyedrop administration. ${ }^{21}$ The UEB differs from other compliance aids in that it allows patients to place eyedrops without reclining their head. While the UEB does not improve accuracy of drop placement, it does lead to less drop wastage and less bottle contamination. The UEB may be well suited for patients who struggle with eyedrop administration.

\section{Acknowledgments}

This work was funded in part by 1 R43EY022515-01A1 (PI: Mosler). Dr Muir receives salary support from a VA HSR\&D Career Development Award.

\section{Disclosure}

The authors report no conflicts of interest in this work.

\section{References}

1. Stone JL, Robin AL, Novack GD, Covert DW, Cagle GD. An objective evaluation of eyedrop instillation in patients with glaucoma. Arch Ophthalmol. 2009;127(6):732-736.

2. Hennessy AL, Katz J, Covert D, et al. A video study of drop instillation in both glaucoma and retina patients with visual impairment. Am J Ophthalmol. 2011;152(6):982-988.

3. Brown MM, Brown GC, Spaeth GL. Improper topical self-administration of ocular medication among patients with glaucoma. Can J Ophthalmol. 1984;19(1):2-5.

4. Gupta R, Patil B, Shah BM, Bali SJ, Mishra SK, Dada T. Evaluating eye drop instillation technique in glaucoma patients. J Glaucoma. 2012; 21(3):189-192.

5. Ostergaard Laursen S, Bjerrum P. Timolol eyedrop-induced severe bronchospasm. Acta Med Scand. 1982;211(6):505-506.

6. Teuchner B, Wagner J, Bechrakis NE, Orth-Holler D, Nagl M. Microbial contamination of glaucoma eyedrops used by patients compared with ocular medications used in the hospital. Medicine (Baltimore). 2015; 94(8):e583. 
7. Geyer O, Bottone EJ, Podos SM, Schumer RA, Asbell PA. Microbial contamination of medications used to treat glaucoma. Br J Ophthalmol. 1995;79(4):376-379.

8. Schein OD, Hibberd PL, Starck T, Baker AS, Kenyon KR. Microbial contamination of in-use ocular medications. Arch Ophthalmol. 1992;110(1):82-85.

9. Schein OD, Wasson PJ, Boruchoff SA, Kenyon KR. Microbial keratitis associated with contaminated ocular medications. Am J Ophthalmol. 1988;105(4):361-365.

10. Alfonso E, Kenyon KR, Ormerod LD, Stevens R, Wagoner MD, Albert DM. Pseudomonas corneoscleritis. Am J Ophthalmol. 1987; 103(1):90-98.

11. Templeton WC 3rd, Eiferman RA, Snyder JW, Melo JC, Raff MJ. Serratia keratitis transmitted by contaminated eyedroppers. Am J Ophthalmol. 1982;93(6):723-726.

12. Solomon A, Chowers I, Raiskup F, Siganos CS, Frucht-Pery J. Inadvertent conjunctival trauma related to contact with drug container tips: a masquerade syndrome. Ophthalmology. 2003;110(4):796-800.

13. Burns E, Mulley GP. Practical problems with eye-drops among elderly ophthalmology outpatients. Age Ageing. 1992;21(3):168-170.

14. Overaker RF, Dodge BC, Epstein DL. A new eyedrop dispensing bottle. Am J Ophthalmol. 1999;128(3):368-370.

15. Sleath B, Blalock S, Covert D, et al. The relationship between glaucoma medication adherence, eye drop technique, and visual field defect severity. Ophthalmology. 2011;118(12):2398-2402.

16. Hennessy AL, Katz J, Covert D, Protzko C, Robin AL. Videotaped evaluation of eyedrop instillation in glaucoma patients with visual impairment or moderate to severe visual field loss. Ophthalmology. 2010;117(12): 2345-2352.
17. Aptel F, Masset H, Burillon C, Robin A, Denis P. The influence of disease severity on quality of eye-drop administration in patients with glaucoma or ocular hypertension. Br J Ophthalmol. 2009;93(5):700-701.

18. Tatham AJ, Sarodia U, Gatrad F, Awan A. Eye drop instillation technique in patients with glaucoma. Eye (Lond). 2013;27(11):1293-1298.

19. Kass MA, Hodapp E, Gordon M, Kolker AE, Goldberg I. Patient administration of eyedrops: observation. Part II. Ann Ophthalmol. 1982; 14(9):889-893.

20. Connor AJ, Severn PS. Force requirements in topical medicine use - the squeezability factor. Eye (Lond). 2011;25(4):466-469.

21. Winfield AJ, Jessiman D, Williams A, Esakowitz L. A study of the causes of non-compliance by patients prescribed eyedrops. Br J Ophthalmol. 1990;74(8):477-480.

22. Hosoda M, Yamabayashi S, Furuta M, Tsukahara S. Do glaucoma patients use eye drops correctly? J Glaucoma. 1995;4(3):202-206.

23. Schwartz GF, Hollander DA, Williams JM. Evaluation of eye drop administration technique in patients with glaucoma or ocular hypertension. Curr Med Res Opin. 2013;29(11):1515-1522.

24. Averns H, Hall J, Webley M. Role of opticare eye drop delivery system in patients with rheumatoid arthritis. J Rheumatol. 1999; 26(12):2615-2618.

25. Nordmann JP, Baudouin C, Bron A, et al. Xal-Ease: impact of an ocular hypotensive delivery device on ease of eyedrop administration, patient compliance, and satisfaction. Eur J Ophthalmol. 2009;19(6) 949-956.
Clinical Ophthalmology

\section{Publish your work in this journal}

Clinical Ophthalmology is an international, peer-reviewed journa covering all subspecialties within ophthalmology. Key topics include: Optometry; Visual science; Pharmacology and drug therapy in eye diseases; Basic Sciences; Primary and Secondary eye care; Patient Safety and Quality of Care Improvements. This journal is indexed on

Submit your manuscript here: http://www.dovepress.com/clinical-ophthalmology-journal

\section{Dovepress}

PubMed Central and CAS, and is the official journal of The Society of Clinical Ophthalmology (SCO). The manuscript management system is completely online and includes a very quick and fair peer-review system, which is all easy to use. Visit http://www.dovepress.com/ testimonials.php to read real quotes from published authors. 